\title{
Gradhiva
}

GRADHIV

Revue d'anthropologie et d'histoire des arts

11 | 2010

Grands hommes vus d'en bas

\section{Exposition « Giinaquq : comme un visage. Masques d'Alaska »}

Château-musée de Boulogne-sur-Mer, Pas-de-Calais (27 juin-7 décembre 2009)

Paz Núñez-Regueiro et André Delpuech

\section{(2) OpenEdition}

Journals

Édition électronique

URL : http://journals.openedition.org/gradhiva/1769

DOI : $10.4000 /$ gradhiva. 1769

ISSN : 1760-849X

Éditeur

Musée du quai Branly Jacques Chirac

Édition imprimée

Date de publication : 19 mai 2010

Pagination : 214-220

ISBN : 978-2-35744-025-8

ISSN : 0764-8928

Référence électronique

Paz Núñez-Regueiro et André Delpuech, « Exposition « Giinaquq : comme un visage. Masques

d'Alaska » », Gradhiva [En ligne], 11 | 2010, mis en ligne le 19 mai 2013, consulté le 20 avril 2019. URL

http://journals.openedition.org/gradhiva/1769; DOI : 10.4000/gradhiva.1769

(c) musée du quai Branly 


\section{EXPOSITION «GIINAOUQ : COMME UN VISAGE. MASOUES D'ALASKA »}

Château-musée de Boulogne-sur-Mer, Pas-de-Calais (27 juin-7 décembre 2009)

Commissariat :

Sven Haakanson Jr, directeur, Alutiiq Museum and

Archaeological Repository, Kodiak, Alaska

Anne-Claire Maury-Laronde, conservateur, château-musée de Boulogne-sur-Mer

Céline Ramio, responsable du service des Collections et du Bâtiment, château-musée de Boulogne-sur-Mer

\section{« Reassurance and awe »}

Dans le livre d'or de l'exposition Giinaquq: like a face, présentée en 2008 à l'Alutiiq Museum de Kodiak, une visiteuse originaire de cet archipel du sud de l'Alaska écrivait ces simples mots : « reassurance and awe», réconfortée, impressionnée et admirative d'avoir pu contempler les masques de ses ancêtres sugpiat, de retour au pays près d'un siècle et demi après leur collecte par un jeune Français de Boulogne-sur-Mer explorant la région. Quelque temps plus tard, le 7 décembre 2009, après six mois de présentation au château-musée de Boulogne-sur-Mer, l'exposition Giinaquq: comme un visage. Masques d'Alaska fermait, forte d'un succès notoire dans la presse et surtout auprès du public, local comme national, avec une fréquentation de près de

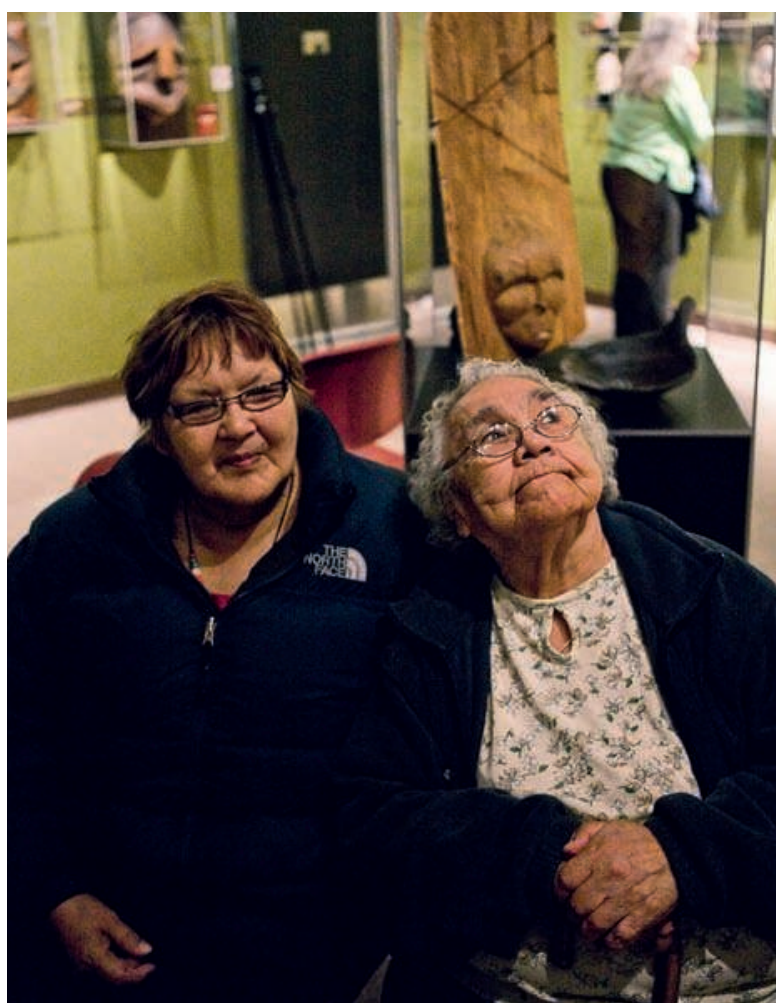

Fig. 1. Vue de l'exposition "Giinaquq, Like a Face», Alutiiq Museum and Archaeological Repository, Kodiak, Alaska @ Koniag Inc., photo Chris Arend.
9000 visiteurs, ce qui en a fait le deuxième événement le plus réussi de ces dernières années pour l'institution boulonnaise. Après l'itinérance alaskienne du projet en 2008, cette manifestation, qui marquait l'aboutissement de la collaboration initiée en 2006 entre le château-musée et l'Alutiiq Museum de Kodiak, présentait une nouvelle fois au public français la collection de masques sugpiat d'Alaska conservée par l'institution boulonnaise, la plus importante au monde. L'exposition se centrait sur le patrimoine matériel collecté par le linguiste Alphonse Pinart (1852-1911) lors de son premier séjour dans le sud de l'Alaska en 1871 , cédé à sa ville natale en 1875 . Cette collection avait été partiellement présentée à Paris en 2002-2003 dans le cadre de l'exposition de préfiguration Kodiak, Alaska, organisée par le musée du quai Branly dans les locaux du musée national des Arts d'Afrique et d'Océanie, peu avant sa fermeture.

Si à Boulogne-sur-Mer les masques restaient au cœur du projet scientifique de la manifestation, ils étaient présentés en dialogue avec l'ensemble du fonds Pinart afin de les replacer dans leur contexte social et historique. Étaient ainsi intégrés à l'exposition, outre les masques, une variété d'objets de la vie quotidienne, des maquettes d'embarcations, des armes, des éléments de parure et des instruments de musique, dont cinq pièces aujourd'hui conservées au musée du quai Branly à Paris. Soit au total près de cent trente objets témoignant de l'étendue de la collecte opérée par le jeune savant boulonnais à un moment crucial de l'histoire du peuple sugpiaq qui, confronté à la domination russe de 1784 à 1867, puis à celle des États-Unis, avait délaissé ses pratiques rituelles ancestrales dans une acculturation progressive à la société russe. À cela s'ajoutaient des pièces archéologiques prêtées par l'Alutiiq Museum de Kodiak et dix-sept masques produits par des artistes sugpiat contemporains, qui permettaient de mettre en évidence la profondeur historique, la permanence dans le temps et la réappropriation d'une tradition artistique vieille de plusieurs siècles.

L'exposition boulonnaise, qui s'organisait en quatre volets répartis sur deux salles, présentait une muséographie simple. L'espace était organisé par des cimaises brunes et vertes que venait ponctuer la collection de masques anciens et modernes, fil conducteur de l'exposition. Cette présentation sobre était égayée par des impressions en grand format de gravures anciennes et de photographies de paysages, par des sérigraphies présentant des citations des cahiers de voyage de Pinart et par des relevés de pétroglyphes anciens retrouvés sur l'île de Kodiak. Regroupés par types, les fameux masques étaient présentés sur un fond noir ou exhibés dans des vitrines transparentes. Ces dernières permettaient d'en apercevoir le revers, évidé ou pas, afin de juger de la portabilité des différents spécimens.

La première section était introduite par une brève présentation de l'expérience alaskienne de Pinart, qui permettait d'expliquer au visiteur la présence à Boulogne-sur-Mer de ce patrimoine unique. La section offrait ensuite un aperçu de la vie quotidienne des Sugpiat au moment de la collecte : transport, alimentation, croyances, chasse à la baleine. Le regroupement des pièces était réalisé de manière thématique. Les masques dont le nom est connu 


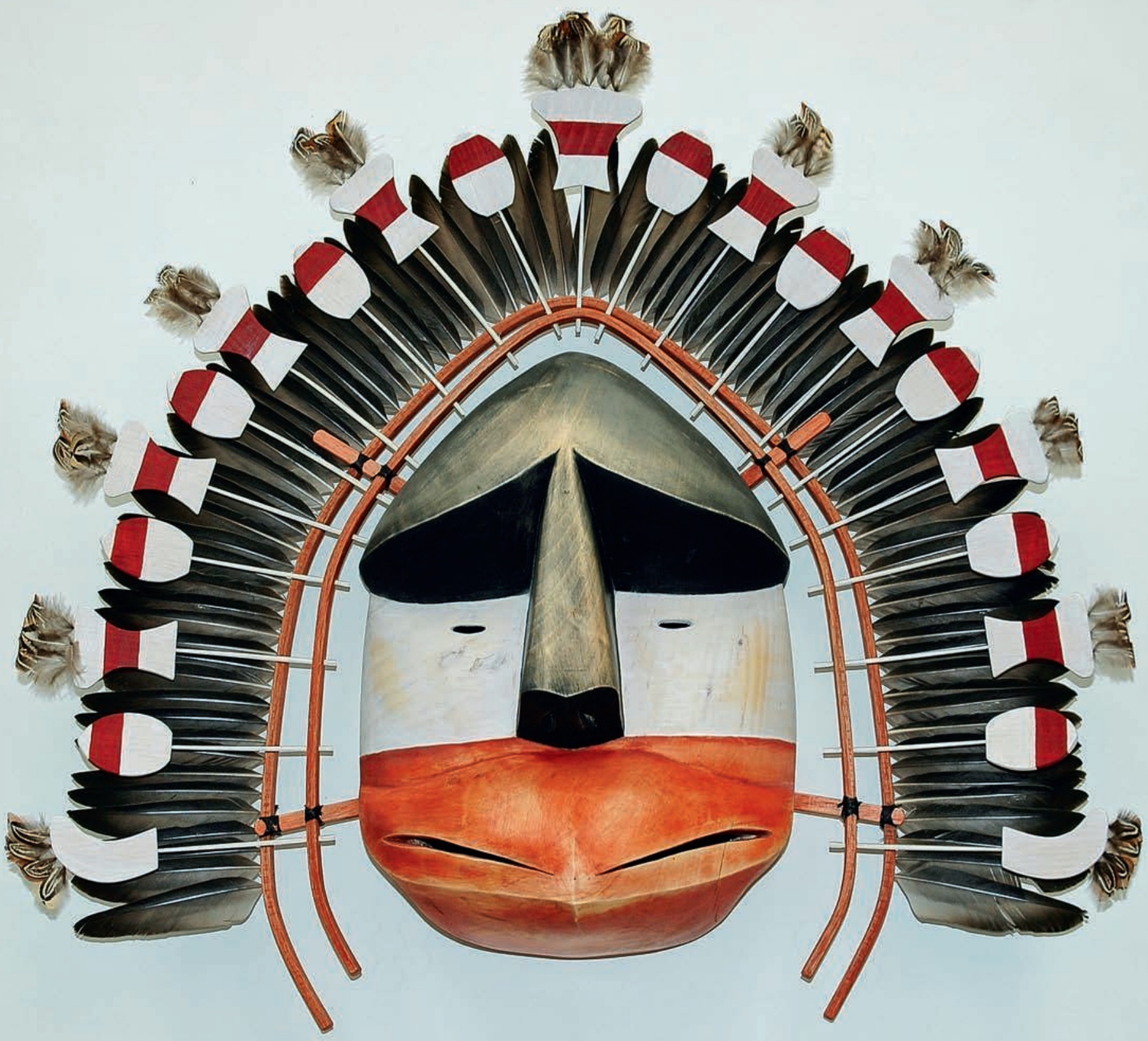

Fig. 2. «L'observateur », Perry Eaton, juillet 2009, bois, peinture à l'huile, plumes ( $57 \mathrm{~cm} \times 58 \mathrm{~cm} \times 17 \mathrm{~cm}$ ) ๑) ville de Boulogne sur mer, photo Jean-Pierre Thomas. 


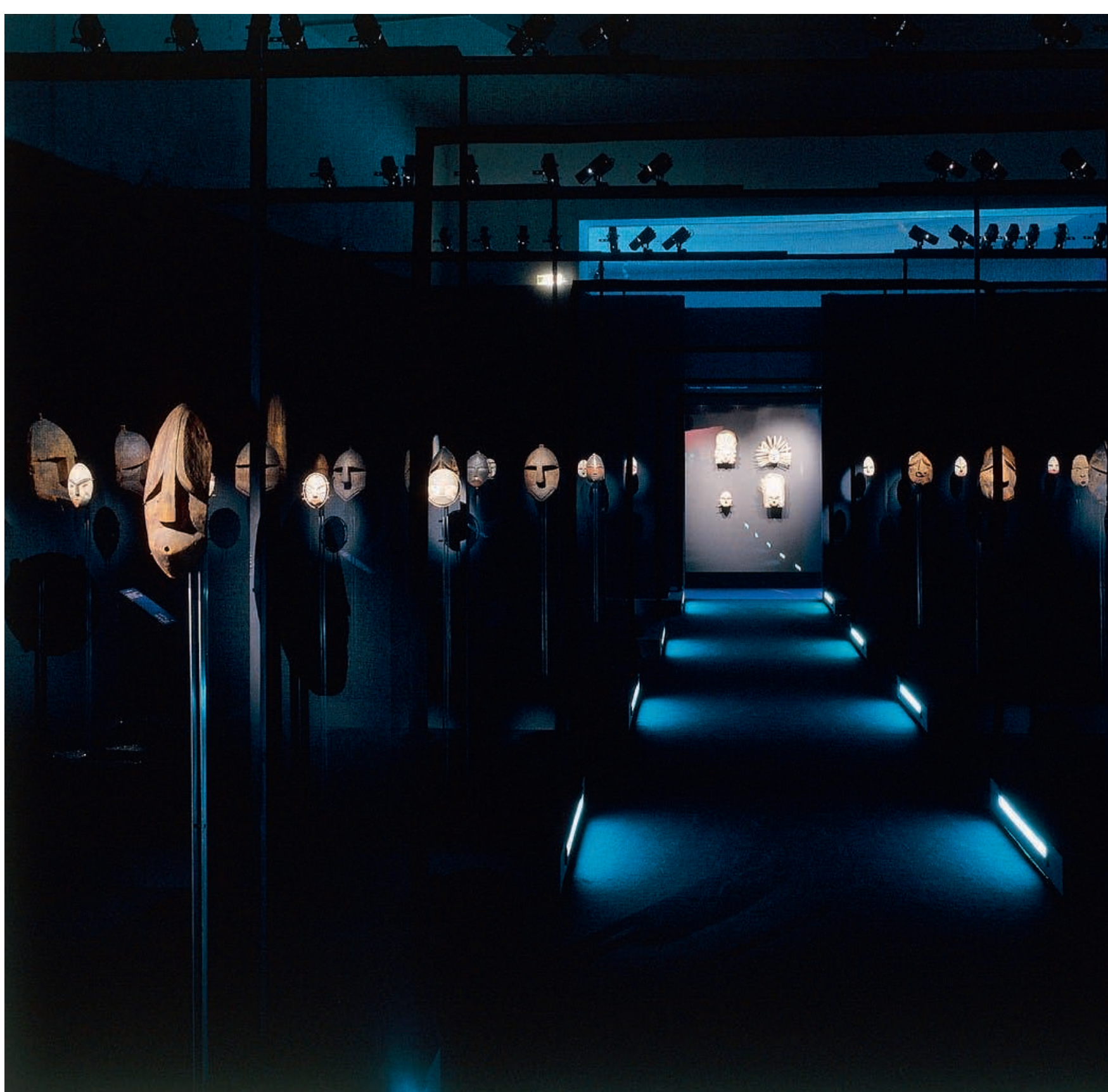

Fig. 3. Vue de l'exposition «Kodiak, Alaska, les masques de la Collection Alphonse Pinart ", Musée National des Arts d'Afrique et d'Océanie, Paris @ musée du quai Branly, photo Nicolas Borel.

étaient associés aux objets de la vie quotidienne que leurs noms évoquent. Par exemple, le masque «porteur de nourriture» était présenté avec des lampes à huile, une cuillère, etc., tandis que le masque «voyageur »était associé à une maquette de l'embarcation appelée angyaq. Par ailleurs, afin de mettre en évidence l'ancienneté de leur production et de leur emploi, les fameux masques masculins et féminins allayak étaient présentés conjointement à des éléments de décor isolés provenant du site archéologique de Karluk One (1400-1750 apr. J.-C.]; de même, d'autres masques étaient exposés en association avec des figurations miniatures en bois de conception formelle similaire, de même provenance archéologique.

Le deuxième volet de l'exposition était consacré aux festivals d'hiver, ces cérémonies de chasse rythmées par les tambours et les chants au cours desquelles les masques étaient employés. Ceux-ci représentaient les esprits animaux et permettaient de faire revivre les êtres qui contrôlaient la vie sur terre, de communiquer avec eux et d'assurer la prospérité de la communauté. 
de diverses analyses. Ainsi, Gaëlle Crenn postula que «malgré le discours défendant l'association des registres ethnologique et esthétique, la dissociation des traitements se li[sai]t très nettement dans l'organisation spatiale de l'exposition », notamment par une «présentation très esthétisante » des masques et une «appréhension esthétique, [excluant] d'autres registres et d'autres voix» (2003: 98-99). Molly Lee et Nelson Graburn soulignèrent quant à eux la place limitée et quelque peu ambiguë accordée au présent dans le traitement de la culture matérielle du passé, tout en saluant le catalogue de l'exposition comme une publication de référence (Lee et Graburn 2003 : 613 et 617).

S'il est tentant, à juste titre, de considérer les expositions de Paris et de Boulogne-sur-Mer comme deux tendances muséographiques opposées, privilégiant l'une le registre esthétique et l'autre celui de l'ethnologie, ce serait cependant oublier que les deux projets s'inscrivent dans le temps à deux moments différents, et qu'ils sont finalement issus l'un de l'autre, bien que conduits par deux équipes aux convictions résolument différentes.

En 2002, la présentation quelque peu spectaculaire de la collection de masques avait beaucoup impressionné les Sugpiat qui avaient fait le déplacement à Paris. Ainsi que le rapporte Perry Eaton, artiste fondateur de l'Alaskan Heritage Center, centre culturel de préservation des savoir-faire et arts alaskiens à Anchorage, ce parti pris très esthétique, d'une grande élégance, qui faisait flotter les masques au-dessus d'un sable noir scintillant dans lequel étaient «plantées » les tiges de soclage des masques, mettait en valeur les pièces et redonnait toute son importance à ce fonds. Alors que le faible niveau d'éclairage de cette salle a pu gêner certains visiteurs occidentaux, il ne choqua pas, au contraire, les visiteurs sugpiat car les masques étaient à l'origine portés et vus dans des habitations closes, éclairées par des lampes à graisse posées à même le sol. Cette manifestation suscita chez les Sugpiat un profond désir de connaissance et de redécouverte de leur propre culture.

Le projet d'exposition au musée du quai Branly avait déjà donné lieu à une prise de contact avec Sven Haakanson, directeur de l'Alutiiq Museum de Kodiak, et l'idée était née de faire voyager les objets en Alaska. Néanmoins, cette idée avait dû être abandonnée, la ville de Boulogne-sur-Mer craignant de se voir confrontée à une demande de rapatriement de ses collections. Ce n'est qu'en 2006, avec l'arrivée à la direction du château-musée d'Anne-Claire Maury-Laronde, conservateur du patrimoine et ethnologue de formation, qu'une nouvelle impulsion fut donnée à cette collaboration franco-alaskienne aux débuts hésitants. Un nouveau projet d'exposition vit alors le jour, qui s'envisageait désormais comme une rencontre des deux communautés autour des collections sugpiat boulonnaises.

À divers égards, la collaboration scientifique qui donna naissance à l'exposition Giinaquq: comme un visage est un modèle de partenariat entre musées occidentaux et communautés d'origine. Le titre de l'exposition, en privilégiant le terme qui désigne le masque en alutiiq, met en exergue la place centrale accordée à la mémoire communautaire. L'étude pluridisciplinaire des masques de la collection Pinart en collaboration directe avec les anciens de Kodiak, la réflexion autour de la légitimité de la conservation en France d'un patrimoine unique pour l'histoire du peuple sugpiaq, et le travail de redécouverte et de réappropriation de ce patrimoine par la communauté sugpiaq actuelle en sont les points les plus marquants. Si l'exposition ne pouvait rendre compte de l'ensemble de ces aspects, la qualité de la préparation scientifique de la présentation boulonnaise permit de redonner son identité à chacun des masques par le déchiffrage, la traduction et l'interprétation des noms inscrits sur leur revers par Pinart, ce qui contribua sur le plan scientifique à une avancée notoire dans la compréhension de leur rôle et de leur signification. Pour les masques dont l'appellation n'est pas parvenue jusqu'à nous, l'attribution d'un nouveau nom par les anciens de Kodiak participa au travail de réappropriation culturelle de ce patrimoine. Ainsi, en décalage avec l'exposition de 2002, dont Crenn a critiqué les «commentaires formulés exclusivement dans un registre esthétique » (2003: 98), chaque masque était ici présenté avec son nom, ancien ou nouveau (suivant une présentation différenciée), en alutiiq et en français. En outre, les masques documentés par Pinart étaient accompagnés par le chant ou le mythe s'y rapportant. Ces textes, déjà publiés dans le catalogue de l'exposition de 2002 par Dominique Desson, firent l'objet d'une réédition: grâce au travail des anciens de la communauté sugpiaq, seuls à maîtriser encore la langue alutiiq, une nouvelle traduction en est proposée dans le catalogue bilingue de l'exposition de 2009.

L'apport boulonnais fut déterminant dans ce travail d'observation, de lecture, de traduction et de discussion autour des masques, offrant un accès inconditionné aux objets, le prêt d'une part importante de la collection Pinart, diverses recherches en archives pour progresser dans la documentation des pièces et la reconstitution de leur parcours après leur arrivée en France, ainsi qu'un investissement financier certain pour la mise en place du partenariat. II convient également de s'arrêter sur la question de la réception par les Sugpiat. La communauté actuelle, loin des «revendications communautaires et de remise en cause du colonialisme » (Crenn 2003 : 94) auxquelles sont souvent confrontés les musées occidentaux, et consciente des aléas de son histoire et des raisons sociales et économiques qui portèrent la communauté sugpiaq à modifier son mode de vie dans le courant du $\mathrm{XIX}^{\mathrm{e}}$ siècle, éprouve en réalité plus de gratitude envers Pinart, qui a su préserver son héritage culturel, que d'aversion pour certaines de ses pratiques de collecte, qui incluent notamment le pillage de sépultures. Certes, ce dernier point n'est pas souligné dans les échanges avec la communauté, tout comme le fait qu'il a également ramené en France des spécimens humains. Ceci dit, loin des craintes de demandes de rapatriement qui avaient en leur temps freiné l'itinérance de l'exposition du musée du quai Branly à Kodiak, c'est un sentiment de gratitude envers la ville de Boulogne-sur-Mer et son personnel qui se dégage des témoignages sugpiat. Cette attitude positive est également le fruit du long travail mené par Haakanson, qui depuis plusieurs années concentre ses efforts afin que les données rapatriées et les événements culturels réalisés à Kodiak et à l'étranger profitent directement 


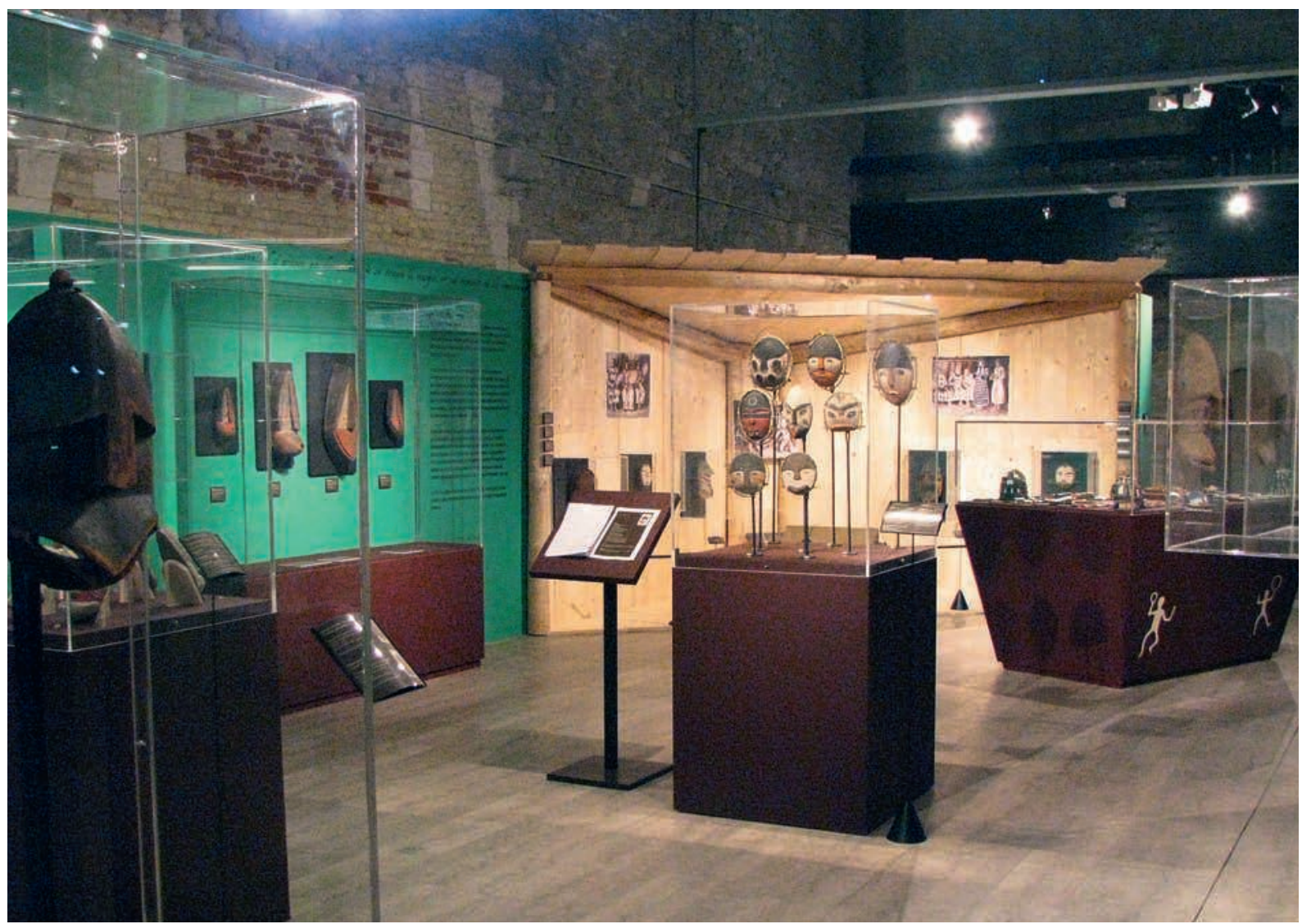

Fig. 4 Vue de l'exposition «Giinaquq, Comme un visage. Masques d'Alaska », Château-musée de Boulogne-sur-Mer ๑) ville de Boulogne sur mer, photo Céline Ramio.

aux membres de la communauté sugpiaq en impliquant ces derniers dans les divers projets orchestrés par l'Alutiiq Museum. Lors de la journée d'études internationale qui accompagna la fin de l'exposition, le 2 décembre 2009, Haakenson exprima très clairement sa démarche, qui ne vise pas à un rapatriement physique des masques mais bien à celui de la connaissance (« repatriation of knowledge »].

La «préfiguration d'une méthode de travail » qui devait être celle du musée du quai Branly, telle qu'elle apparaît annoncée par les ministres de l'époque dans le catalogue de l'exposition (Désveaux 2002: 12), impliquait certes une collaboration avec le châteaumusée de Boulogne-sur-Mer, les musées de Saint-Pétersbourg, le musée de l'Homme et le Muséum national d'histoire naturelle, mais omettait maladroitement de faire valoir la participation de l'Alutiiq Museum au projet en la personne d'Haakanson, sollicité par Emmanuel Désveaux, commissaire de l'exposition et directeur du projet pour l'enseignement et la recherche du musée du quai Branly de l'époque. Le travail mené par ce dernier, qui privilégia l'échange avec son homologue sugpiaq, anthropologue comme lui, mais aussi ardent défenseur du renouveau culturel et spirituel de la communauté alutiiq en Alaska, n'aboutit pas cependant sur un ensemble combinant les approches des divers spécialistes impliqués dans le projet sur la fonction et la symbolique des masques, et moins encore sur un échange avec la communauté concernée. L'important travail réalisé à l'époque pour rassembler les œuvres des rares institutions nationales détentrices d'objets sugpiat du xix ${ }^{\mathrm{e}}$ siècle et les contributions des spécialistes dans le domaine ne permit pas, dans le temps imparti, une réelle synthèse des données disponibles. Certes, les objectifs de l'exposition de 2002 étaient ailleurs. Ainsi que le soulignait clairement Stéphane Martin, président-directeur général du musée du quai Branly, le but de l'exposition de masques était « d'en chercher le sens, d'en retrouver les clés essentielles, de décrire, sous la forme artistique du masque, les mythes et les rites d'une société disparue » (ibid.). Le partenariat mis en place entre Boulogne-sur-Mer et Kodiak comme l'exposition qui en découla avaient quant à eux pour principal objectif de recontextualiser les masques et de montrer la vivacité retrouvée d'une culture qui s'était censurée et vue oubliée pendant près d'un siècle et demi.

En ce sens, l'exposition du château-musée est une réussite : claire, riche en informations et donnant la parole aux acteurs de la communauté sugpiaq. Dans cette présentation exhaustive de la collection Pinart et de ses masques, et dans le cadre d'une exposition plaçant pertinemment la culture matérielle du xIx ${ }^{\mathrm{e}}$ siècle dans la droite ligne de productions vieilles de plusieurs siècles, un point aurait cependant mérité d'être développé (comme il l'est d'ailleurs dans le catalogue par Haakanson et Steffian 
[2009: 1-10]] : la contextualisation du patrimoine archéologique présenté. Peu était dit, en fin de compte, au sujet des données retrouvées sur place, à Kodiak, dont certains éléments surprenants comme les masques miniatures constituaient l'un des points forts de l'exposition: quels enseignements en tirer sur les anciens habitants de Kodiak et sur la profondeur historique de cette société?

De même, la section d'art contemporain aurait pu être davantage mise en valeur par une présentation plus détaillée de chacune des œuvres présentées. Celles-ci, très différentes formellement et conceptuellement, constituent un vecteur privilégié pour comprendre comment se met en place cette réappropriation de la culture sugpiaq par des artistes confirmés mais aussi par de jeunes apprentis, dans une assimilation participative qui adopte des formes multiples: influence directe de certains masques de la collection Pinart (Les Quatre Vieux Hommes, Perry Eaton, 2002 2003, coll. Alutiiq Museum) et des événements politiques contemporains comme la guerre en Irak (De peur que nous oubliions, Sven Haakanson Jr, 2007, coll. Alutiiq Museum], emploi traditionnel de matériaux que l'on trouve sur la plage - ce qui, au xxle siècle, signifie créer avec des morceaux de bouée et autres déchets (Masque phoque, Lena Snow Amason, 2009, coll. de l'artiste) -, etc. Cette création encore très mal connue demande à être analysée et mieux diffusée, et il est encourageant de voir qu'un musée français a une politique d'acquisition dans ce domaine.

Pour ceux qui n'ont pu faire le déplacement à Boulogne-sur-Mer, il reste le très complet catalogue bilingue anglais-français de l'exposition (Haakanson Jr et Steffian 2009, 280 pages, 375 photographies], permettant une meilleure compréhension de la culture sugpiaq, qui reste l'une des moins connues d'Alaska. On y trouve : une synthèse sur les masques archéologiques, leurs matériaux et leur mode de fabrication; une description de la collecte de Pinart et de I'histoire des pièces depuis leur arrivée en France; une présentation historique des villages sugpiat de Kodiak au xıx ${ }^{e}$ siècle; un catalogue raisonné de la collection de masques, assorti de nombreuses prises de vue de chaque pièce; enfin, le point de vue de l'artiste Perry Eaton sur la valeur et le rôle de la collection Pinart aujourd'hui.

Des annexes compilent les chants des masques enregistrés par Pinart et de très nombreuses illustrations complètent la publication: photographies historiques, gravures, prises de vue des différentes étapes du projet Giinaquq, etc. À ce propos, saluons le parti pris de l'ouvrage, qui présente chaque masque sous plusieurs angles: de face, de profil, de dos, avec des gros plans sur des détails techniques ou des décors. La taille, l'épaisseur des objets, leur gravure, les modes de fixation, etc. en deviennent beaucoup plus lisibles pour un public qui n'a pas accès aux originaux. II est ainsi intéressant d'observer que les artistes sugpiat contemporains, qui n'avaient auparavant accès qu'aux seules photographies de face illustrant les publications antérieures, dont le catalogue de 2002, créaient des œuvres sans profondeur, presque à plat.

Malgré son éloignement géographique, la collection conservée à Boulogne-sur-Mer a donc su déclencher et être le catalyseur d'un intérêt accru pour ce fonds de la part des deux communautés et d'un renouveau culturel et artistique sans précédent à Kodiak. Force est de constater que le «dialogue des cultures» a finalement lieu entre Sugpiat et Boulonnais.

$$
\begin{array}{r}
\text { Paz Núñez-Regueiro, musée du quai Branly } \\
\text { pnr@quaibranly.fr } \\
\text { André Delpuech, musée du quai Branly } \\
\text { adı@quaibranly.fr }
\end{array}
$$

\section{Bibliographie}

\section{CRENN, Gaëlle}

2003 « Rhétorique de la transparence et légitimité muséale : à propos de trois expositions d'ethnologie », Quaderni 52(1) : 93-103.

\section{DésveAux, Emmanuel (dir.)}

2002 Kodiak, Alaska. Les masques de la collection Alphonse Pinart. Paris, Adam Biro-musée du quai Branly.

HaAkanson, Sven Jr et Stefrian, Amy (dir.)

2009 Giinaquq : like a face, comme un visage. Sugpiaq Masks from the Kodiak Archipelago. Fairbanks, University of Alaska Press.

\section{Lee, Molly et Graburn, Nelson}

2003 « Reconfiguring Kodiak: the past and the present in the present », American Anthropologist 105(3) : 613-620.

\section{Prince, Robert}

2009 Finding their own dance: reawakening the alaskan alutiiq arts, documentaire réalisé par Robert Prince, de l'université de Fairbanks, Alaska, et produit par Ellen Van't Hof, du Calvin College de Grand Rapids, Michigan (http://alutiiqdancefilm.org/index.html). 